\title{
Zero-Shear Viscosity of Branched Polymer Solutions
}

\author{
Yoshiaki TAKahashi, Fumitoshi SuzuKI, Masahiko MIYACHI, \\ Ichiro NoDA, and Mitsuru NAGASAWA \\ Department of Synthetic Chemistry, Nagoya University, \\ Chikusa-ku, Nagoya 464, Japan
}

(Received August 5, 1985)

\begin{abstract}
The zero-shear viscosity $\eta^{\circ}$ of star-shaped polymers with different numbers of branches in good solvents were analyzed using the scaling theory of de Gennes. It was concluded that the semidilute region exists in the viscosity behavior of branched polymers if the molecular weight of branches are high enough. The scaling theory agrees with experimental data of the branched polymers if we recognize that the molecular weight dependence of zero-shear viscosity is higher for branched polymers than for linear polymers.

KEY WORDS Star-Shaped Polymer / Comb-Shaped Polymer / Branched Polymer / Zero-Shear Viscosity / Semidilute Solution / Scaling Theory /
\end{abstract}

The viscosity behavior of linear polymer solutions in a wide range of polymer concentration is well understood ${ }^{1,2}$ if we take into account the semidilute region proposed by de Gennes. ${ }^{3}$ In semidilute solutions, a viscosity parameter $\eta_{\mathrm{R}}^{0}$ defined by $\eta_{\mathrm{R}}^{0} \equiv \eta_{\mathrm{sp}}^{0} / C[\eta]$, where $\eta_{\mathrm{sp}}^{0}=\left(\eta^{0}-\eta_{\mathrm{s}}\right) / \eta_{\mathrm{s}}, \eta_{\mathrm{s}}$ being the solvent viscosity, and $[\eta]$ is the intrinsic viscosity, is given by

$$
\eta_{\mathrm{R}}^{0} \propto\left(C / C^{*}\right)^{(a+1-3 v) /(3 v-1)}
$$

or

$$
\eta_{\mathrm{sp}}^{0} / M^{a} \propto C^{a /(3 v-1)}
$$

where $v$ is the excluded volume exponent defined as $\left\langle s^{2}\right\rangle \propto M^{2 v}$ and $C^{*}=3 M / 4 \pi\left\langle s^{2}\right\rangle^{3 / 2} N_{\mathrm{A}}$ is the critical concentration at which polymer coils begin to overlap each other, $\left\langle s^{2}\right\rangle$ is the radius of gyration, and $N_{\mathrm{A}}$ is Avogadro's number. Moreover, it can be assumed for linear polymers ${ }^{1,2}$ that $C / C^{*}$ is proportional to $C[\eta]$. Since $[\eta] \propto\left\langle s^{2}\right\rangle^{3 / 2} / M$ holds for branched polymers, ${ }^{4} C / C^{*}$ for branched polymers may also be proportional to $C[\eta]$.

If the polymer concentration is lower than a critical value of $C / C^{*}$, i.e., in dilute solutions, $\eta_{\mathrm{R}}^{0}$ is given by
$\eta_{\mathrm{R}}^{0}=1+k^{\prime}[\eta] C+\cdots=1+k\left(C / C^{*}\right)+\cdots$

where $k=3 k^{\prime} \Phi / 4 \pi N_{\mathrm{A}}, \quad k^{\prime}$ and $\Phi$ are the Huggins' constant and the Flory coefficient concerning intrinsic viscosity, respectively. If the polymer concentration is higher than a critical concentration $C^{* *}$, i.e., in concentrated solutions, $\eta_{\mathrm{R}}^{0}$ cannot be expressed as a function of $C / C^{*}$ and $\eta_{\mathrm{sp}}^{0} / M^{\mathrm{a}}$ increase with concentration with a power higher than in eq 2 .

Equations 1 and 2 were well supported by experiments $^{1,2}$ for linear polymers if the molecular weights of polymers are high enough. As the polymer concentration is increased, $\eta^{0}$ of linear polymer solutions crossovers from the dilute solution behavior to the concentrated solution behavior via the semidilute solution. If the molecular weight is low, however, the semidilute region is not observed and $\eta^{0}$ of the polymer solution crossovers from the dilute solution behavior directly to the concentrated solution behavior.

Although the radius of gyration of a branched polymer is much smaller than that of the corresponding linear polymer with 
the same molecular weight and, hence, $C^{*}$ for branched polymers is much higher than that of linear polymers, the relationship (1) and (2) are expected to hold for branched polymer solutions if the molecular weights of branches are high enough. The aim of this work is to examine this speculation.

\section{EXPERIMENTAL}

\section{Samples and Solvents}

The polymer samples used are three-armed, Star-shaped poly $(\alpha$-methylstyrenes) (P $\alpha \mathrm{MS})$ (Sample code S-22) and highly branched comb-shaped polystyrenes (PS) (Sample code $\mathrm{H}-8, \mathrm{I}-6, \mathrm{~J}-2$ ). The latter may also be regarded as star-shaped polymers since the molecular weight of the backbone is lower than that of branches. ${ }^{5}$ These series of polymers were anionically prepared ${ }^{6,7}$ and have narrow molecular weight distributions in branches and backbone. However, the branched PS samples have distributions in number of branches per molecule, though they were carefully fractionated. Their GPC chromatograms obtained by a Toyo Soda high speed liquid chromatograph HLC-802A with resolution columns GMHXL $\times 2$ are shown in Figure 1 .

The molecular characteristics of these samples and of other branched samples discussed in this work are listed in Table I. Sample S-26 is a three-armed, star-shaped $\mathrm{P} \alpha \mathrm{MS}$, while CS series are four-armed, starshaped PS. Although the mean numbers of branches of three comb-shaped samples are not exactly identical, they may be regarded as a series of samples differing only in length of branch, since the viscosity of highly branched polymers are insensitive to the number of branches per molecule. $^{6}$

Two kinds of good solvent, $\alpha$-chloronaphthalene $(\alpha-\mathrm{CN})$ and toluene, were used for PS, while only toluene was used for P $\alpha$ MS. The purification methods and physical properties of these solvents were reported previously. ${ }^{8,9}$

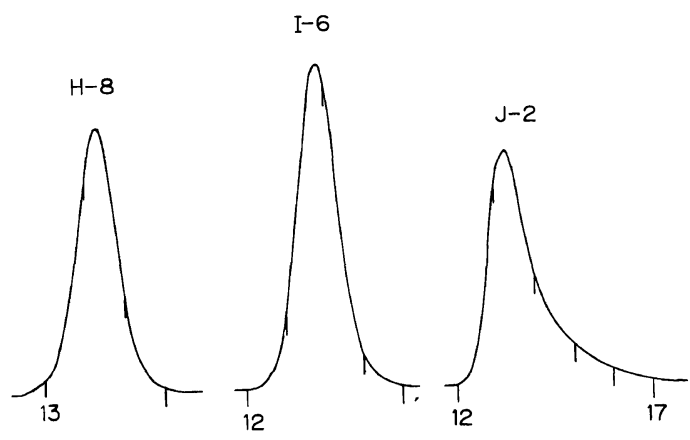

Figure 1. GPC chromatograms of samples H-8, I-6, and J-2. Solvent, tetrahydrofuran $+5 \%$ tetramethylenediamine. Polymer concentration, ca. $0.2 \mathrm{~g} \mathrm{dl}^{-1}$.

Table I. Sample characteristics

\begin{tabular}{|c|c|c|c|c|c|}
\hline \multirow{2}{*}{$\begin{array}{c}\text { Sample } \\
\text { code }\end{array}$} & \multicolumn{2}{|c|}{ Molecular weight } & \multirow{2}{*}{$f^{a}$} & \multirow{2}{*}{$\frac{[\eta]}{\mathrm{dlg}^{-1}}$} & \multirow{2}{*}{$\frac{C^{*} / \mathrm{g} \mathrm{ml}^{-1}}{\times 10^{-2}}$} \\
\hline & $M_{w} \times 10^{-6}$ & $M_{n} \times 10^{-6}$ & & & \\
\hline S-22 & 0.90 & & 3 & $1.4_{1}$ & $0.63_{3}^{b}$ \\
\hline S-26 & 3.60 & & 3 & $4.2_{2}$ & $0.28_{1}^{b}$ \\
\hline $\mathrm{H}-8^{\mathrm{c}}$ & $1.4_{0}$ & & 10.1 & 1.29 & \\
\hline$I-6^{c}$ & $2.4_{4}$ & & 13.3 & $1.6_{6}$ & \\
\hline$J-2^{c}$ & $4.7_{7}$ & & 13.0 & $2.8_{0}$ & \\
\hline CS-25 & & $0.28_{0}$ & 4 & & \\
\hline $\mathrm{CS}-60$ & & $0.48_{8}$ & 4 & & \\
\hline CS-81 & $0.76_{0}$ & $0.75_{0}$ & 4 & & \\
\hline
\end{tabular}

${ }^{\text {a }} f$ is the number of branches per molecule.

b Reported in ref 12 .

c The number average molecular weight of backbone is $9.7_{6} \times 10^{4}$.

\section{Measurements}

The original solutions were prepared by mixing weighed amounts of polymer and solvent at about $50^{\circ} \mathrm{C}$ and were gently stirred a few times a day until they became uniform. The solutions with lower concentrations were prepared by diluting the original solutions by weight. Polymer concentrations were converted to $\mathrm{g} \mathrm{ml}^{-1}$ assuming the additivity of specific volumes of polymer and solvent.

The zero-shear viscosity $\eta^{0}$ of $\alpha-\mathrm{CN}$ solutions of comb-shaped PS were measured by a Weissenberg rheogoniometer R-17 of Sangamo Controls Ltd., equipped with a gapservo system at $50.0 \pm 0.1^{\circ} \mathrm{C}$. The cone and plate with $5 \mathrm{~cm}$ diameter and $4^{\circ}$ angle were 
Zero-Shear Viscosity of Branched Polymer Solutions

Table II. Zero-shear viscosity

\begin{tabular}{|c|c|c|c|c|c|c|c|c|c|}
\hline \multirow{2}{*}{ Sample } & \multirow{2}{*}{ Solvent } & \multirow{2}{*}{$\frac{\text { Temp }^{\mathbf{a}}}{{ }^{\circ} \mathrm{C}}$} & \multirow{2}{*}{$\frac{\mathrm{C} \times 10^{2}}{\mathrm{gcm}^{-3}}$} & \multirow{2}{*}{$\frac{\eta^{\circ}}{\mathrm{cP}}$} & \multirow{2}{*}{ Sample } & \multirow{2}{*}{ Solvent } & \multirow{2}{*}{$\frac{\mathrm{Temp}^{\mathrm{a}}}{{ }^{\circ} \mathrm{C}}$} & \multirow{2}{*}{$\frac{\mathrm{C} \times 10^{2}}{\mathrm{gcm}^{-3}}$} & \multirow{2}{*}{$\frac{\eta^{\circ}}{\mathrm{cP}}$} \\
\hline & & & & & & & & & \\
\hline \multirow[t]{11}{*}{ S-22 } & \multirow[t]{11}{*}{ Toluene } & \multirow[t]{11}{*}{30.0} & 7.02 & $4.64 \times 10^{1}$ & \multirow[t]{19}{*}{ I-6 } & \multirow[t]{4}{*}{$\alpha-\mathrm{CN}$} & \multirow[t]{4}{*}{50.0} & 30.6 & $1.32 \times 10^{5}$ \\
\hline & & & 6.34 & $3.47 \times 10^{1}$ & & & & 27.2 & $6.36 \times 10^{4}$ \\
\hline & & & 5.02 & $2.07 \times 10^{1}$ & & & & 23.1 & $1.82 \times 10^{4}$ \\
\hline & & & 4.34 & $1.41 \times 10^{1}$ & & & & 19.9 & $9.21 \times 10^{3}$ \\
\hline & & & 3.46 & $8.51 \times 10^{0}$ & & \multirow[t]{7}{*}{ Toluene } & \multirow[t]{7}{*}{30.0} & 19.6 & $2.17 \times 10^{3}$ \\
\hline & & & 2.59 & $5.26 \times 10^{0}$ & & & & 16.1 & $9.34 \times 10^{2}$ \\
\hline & & & 1.72 & $2.94 \times 10^{0}$ & & & & 9.80 & $1.66 \times 10^{2}$ \\
\hline & & & 1.12 & $1.71 \times 10^{0}$ & & & & 6.98 & $6.39 \times 10^{1}$ \\
\hline & & & 0.859 & $1.43 \times 10^{0}$ & & & & 5.52 & $3.77 \times 10^{1}$ \\
\hline & & & 0.601 & $1.09 \times 10^{0}$ & & & & 3.84 & $1.78 \times 10^{1}$ \\
\hline & & & & & & & & 2.63 & $1.04 \times 10^{1}$ \\
\hline \multirow[t]{21}{*}{$\mathrm{H}-8$} & \multirow[t]{2}{*}{$\alpha-\mathrm{CN}$} & \multirow[t]{2}{*}{50.0} & 30.6 & $3.92 \times 10^{4}$ & & & & 1.80 & $5.27 \times 10^{0}$ \\
\hline & & & 24.5 & $1.14 \times 10^{3}$ & & & & 1.20 & $2.96 \times 10^{0}$ \\
\hline & \multirow[t]{19}{*}{ Toluene } & \multirow[t]{19}{*}{30.0} & 18.3 & $5.66 \times 10^{2}$ & & & & 1.01 & $2.46 \times 10^{0}$ \\
\hline & & & 15.1 & $2.88 \times 10^{2}$ & & & & 0.697 & $1.60 \times 10^{0}$ \\
\hline & & & 12.2 & $1.40 \times 10^{2}$ & & & & 0.507 & $1.23 \times 10^{0}$ \\
\hline & & & 9.67 & $7.31 \times 10^{1}$ & & & & 0.447 & $1.13 \times 10^{0}$ \\
\hline & & & 7.67 & $4.26 \times 10^{1}$ & & & & 0.278 & $8.51 \times 10^{-1}$ \\
\hline & & & 6.09 & $2.54 \times 10^{1}$ & & & & & \\
\hline & & & 4.84 & $1.65 \times 10^{1}$ & \multirow[t]{13}{*}{$\mathrm{J}-2$} & \multirow[t]{4}{*}{$\alpha-\mathrm{CN}$} & \multirow[t]{4}{*}{50.0} & 20.0 & $1.32 \times 10^{5}$ \\
\hline & & & 3.84 & $1.09 \times 10^{1}$ & & & & 16.1 & $4.14 \times 10^{4}$ \\
\hline & & & 3.05 & $7.36 \times 10^{0}$ & & & & 12.8 & $1.34 \times 10^{4}$ \\
\hline & & & 2.42 & $5.26 \times 10^{0}$ & & & & 10.4 & $5.14 \times 10^{3}$ \\
\hline & & & 1.93 & $3.81 \times 10^{0}$ & & \multirow[t]{9}{*}{ Toluene } & 30.0 & 5.05 & $9.75 \times 10^{1}$ \\
\hline & & & 1.53 & $2.82 \times 10^{0}$ & & & & 3.48 & $3.74 \times 10^{1}$ \\
\hline & & & 1.22 & $2.14 \times 10^{0}$ & & & & 2.57 & $1.95 \times 10^{1}$ \\
\hline & & & 0.962 & $1.65 \times 10^{0}$ & & & & 1.60 & $8.00 \times 10^{0}$ \\
\hline & & & 0.767 & $1.34 \times 10^{0}$ & & & & 1.13 & $4.45 \times 10^{0}$ \\
\hline & & & & & & & & 0.704 & $2.37 \times 10^{0}$ \\
\hline & & & & & & & & 0.549 & $1.95 \times 10^{0}$ \\
\hline & & & & & & & & 0.374 & $1.36 \times 10^{0}$ \\
\hline & & & & & & & & 0.195 & $9.16 \times 10^{-1}$ \\
\hline
\end{tabular}

a $\pm 0.1^{\circ} \mathrm{C}$.

used. The measurements in toluene solutions were carried out by a capillary viscometers of the Maron-Krieger-Sisko type or a modified Ubbelohde type at $30.0^{\circ} \mathrm{C}$. The values of $[\eta]$ in toluene are listed in Table I.

\section{RESULTS AND DISCUSSION}

The zero-shear viscosity $\eta^{0}$ of comb-shaped PS in $\alpha-C N$ and in toluene and also $\eta^{0}$ of threearmed, star-shaped $\mathrm{P} \alpha \mathrm{MS}$, obtained in this work, are listed in Table II and plotted in Figure 2 in the form of $\log \eta_{\mathrm{sp}}^{0} v s . \log C$. The zero-shear viscosity $\eta^{0}$ of three-armed (S-22 and S-26) and four-armed (CS-25, CS-60, and CS-81), star-shaped polymers in $\alpha-\mathrm{CN}$ were previously reported by Kajiura et al. ${ }^{10}$ and Isono et al., ${ }^{5}$ respectively. To compare the viscosity data with the scaling theory (eq 1), informations concerning the exponent $a$ in the relationship $\eta^{0} \propto M^{a}$, the excluded volume exponent $v$ and the critical concentration $C^{*}$ are needed.

The molecular weight dependence of $\eta^{0}$ of branched polymers has not yet been fully studied. It was reported that the exponent $a$ in 


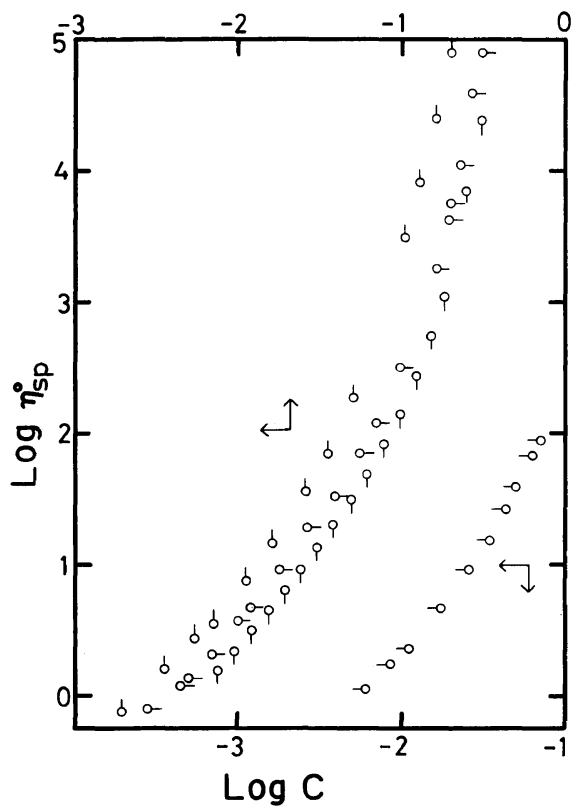

Figure 2. Polymer concentration dependence of zeroshear specific viscosity $\eta_{\mathrm{sp}}^{\circ}$ in good solvents $(\alpha-\mathrm{CN}$ and toleune). Symbols $\mathrm{O}, \mathrm{O}^{-}, \mathrm{O}$, and $-\mathrm{O}$ denote the data for J-2, I-6, H-8, and S-22, respectively.

$\eta^{0} \propto M^{a}$ relationship for star-shaped polybutadienes at melt states ${ }^{11,12}$ and star-shaped polyisoprenes in concentrated solutions ${ }^{13}$ increases with molecular weight. On the other hand, the value of $a$ for four-armed, starshaped PS at melt states ${ }^{14}$ and in concentrated solutions $^{15}$ is $4.1-4.5$ independent of molecular weight in the molecular weight ranges studied here. In the case of four-armed, starshaped PS used in the present work as well as in the case of comb-shaped PS having a constant degree of branching, it was reported that the exponent $a$ is 4.3 at melt states. ${ }^{6,16}$ If the molecular weight of a branch is not high, the branched polymers cannot form entanglements fully in solution and, consequently, the exponent $a$ may be found lower than 4.3. ${ }^{16}$ Even in such cases, it was pointed out that the value of $a$ approaches 4.3 as the molecular weight increases. ${ }^{16}$ In this paper, therefore, it is assumed that the exponent $a$ for both combshaped and four-armed, star-shaped PS in

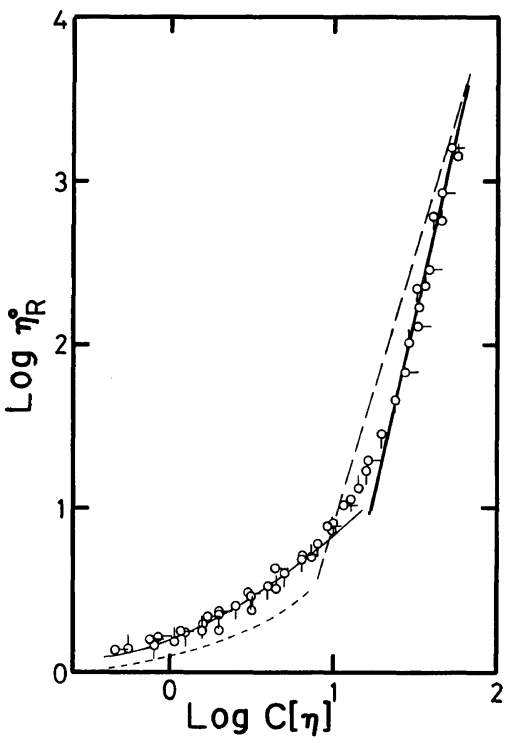

Figure 3. Double logarithmic plots of reduced viscosity $\eta_{\mathrm{R}}^{\circ} v s$. degree of coil overlapping $C[\eta]$ for combshaped PS in good solvents ( $\alpha-\mathrm{CN}$ and toluene). Symbols are the same as in Figure 2. The thin solid line denotes the calculated values of eq. (3) $\left(k^{\prime}=0.6\right)$, while the thick solid line denotes the theoretical slope of eq $1(a=4.3$, $\left.v=0.59_{5}\right)$. The dotted and broken lines denote the corresponding data for linear PS in good solvents. ${ }^{2}$

solution is 4.3 if the molecules are fully entangled. The assumption seems to be reasonable since it is well known that the molecular weight dependence of $\eta^{0}$ of linear flexible polymers in solution is the same as that at melt states if the polymers are fully entangled. ${ }^{17}$ However, the molecular weight dependence of $\eta^{0}$ of three-armed, star-shaped P $\alpha$ MS is not known. The exponent $a$ for this sample may be inbetween 3.4 and 4.3.

From previous works ${ }^{4,18}$ using the same samples, we may safely assume that the excluded volume exponent $v$ for the present branched polymers in good solvents is equal to the value for linear polymers $\left(v=0.59_{5}\right.$ for PS, $0.58_{5}$ for $\left.\mathrm{P} \alpha \mathrm{MS}\right)$. Since it is difficult to determine $C^{*}$ unambiguously for comb-shaped PS, which have distributions in the degree of branching, moreover, we use $C[\eta]$ instead of $C / C^{*}$ as the degree of coil overlapping.

The data of comb-shaped PS in Figure 2 are 


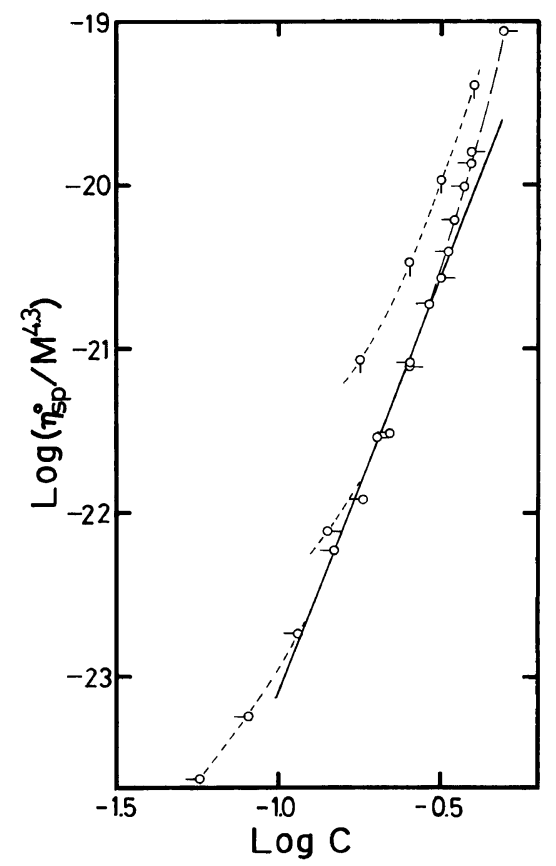

Figure 4. Plots of $\log \eta_{\mathrm{sp}}^{\circ} / M^{4.3} v s . \log C$ for four-armed star-shaped PS in $\alpha-\mathrm{CN}^{5}$ Symbols $\bigcirc, \bigcirc-$, and $-\bigcirc$ denote the data for CS-25, CS-60, and CS-81, respectively. The solid line denotes the slope calculated from eq $2\left(a=4.3, v=0.59_{5}\right)$.

replotted in the form of $\log \eta_{\mathrm{R}}^{0} v s . \log C[\eta]$ in Figure 3. All data seem to compose a universal line as predicted from eq 1 and 3 . The thick and thin solid lines in Figure 3 show the calculated values of eq 1 and 3, respectively, whereas the broken and dotted lines denote the corresponding data of linear PS in good solvents. In $\log C[\eta]>1.4, \eta_{\mathrm{R}}^{0}$ of branched PS fit the thick solid line with a slope calculated from eq 1 , assuming $a=4.3$ and $v=0.59_{5}$.

In the dilute region $(C[\eta]<10), \quad \eta_{\mathrm{R}}^{0}$ of branched PS are higher than those of linear PS. The difference is caused by the higher value of Huggins' constant $k^{\prime}$ for branched PS than for linear PS $\left(k^{\prime}=0.35\right.$ for linear PS, while $K^{\prime} \simeq 0.6$ for branched PS). With increasing $C[\eta]$, the data of branched PS become lower than those of linear PS but show higher dependence on $C[\eta]$.

The data of four-armed, star-shaped PS (CS

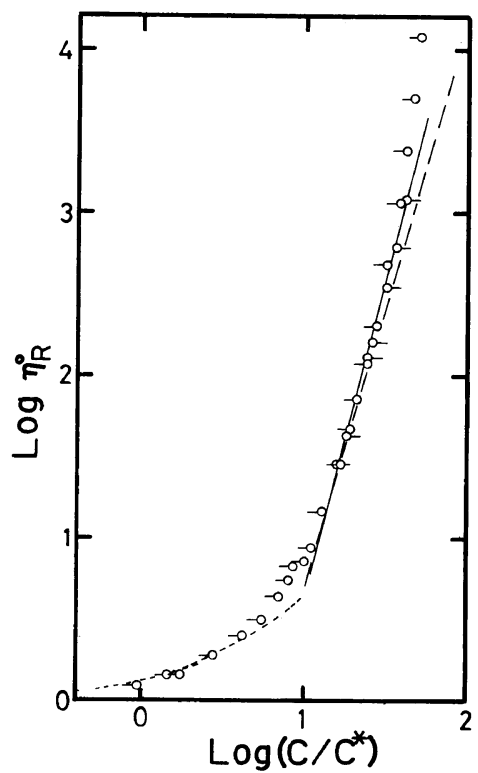

Figure 5. Plots of $\log \eta_{\mathrm{R}}^{\circ} v s . \log \left(C / C^{*}\right)$ for three-armed star-shaped $\mathrm{P} \alpha \mathrm{MS}$ in good solvents $\left(\alpha-\mathrm{CN}^{10}\right.$ and toluene). Symbols $-\mathrm{O}$ and $\bigcirc-$ denote the data for $\mathrm{S}-22$ and S-26, respectively. The dotted and broken lines denote the data for linear $\mathrm{P} \alpha \mathrm{MS}$ in good solvents. ${ }^{1}$ The solid line denote the slope calculated from eq $1\left(a=3.7, v=0.58_{5}\right)$.

series) in $\alpha-\mathrm{CN}$ reported by Isono et $a .^{5}$ cannot be compared with eq 1 since the critical concentrations $\left(C^{*}\right.$ or $\left.[\eta]\right)$ of these samples are not known. However, knowing the molecular weight dependence of $\eta^{0}(a=4.3)$ the data can be compared with eq 2 by plotting the data in the form of $\log \eta_{\mathrm{sp}}^{0} / M^{4.3} v s . \log C$, as shown in Figure 4. In this kind of plot, the crossover between the dilute region and the semidilute region is not clear, particularly since the experimental points in the dilute solution are scarce. Nevertheless, it can be pointed out that the data of $\log \eta_{\mathrm{sp}}^{0} / M^{4.3}$ approaches a straight line with the slope of eq 2 (assuming $a=4.3$ and $\left.v=0.59_{5}\right)$ as the concentration increases.

If the polymer concentration becomes higher than $C \simeq 0.3 \mathrm{~g} \mathrm{ml}^{-1}, \eta_{\mathrm{sp}}^{0} / M^{4.3}$ again deviates from the theoretical line. The deviation from the theoretical line occurs since the solution enter into the concentrated region where the excluded volume effect is screened and the 


\section{Y. TAKAHASHI $e t$ al.}

local frictional coefficient of segment may change with concentration, as reported for linear polymers. ${ }^{1}$ Moreover, the data of the lowest molecular weight sample (CS-25) do not converge on the theoretical line but crossover from the dilute solution directly to the concentrated solution. The polymer molecules may not be fully entangled in this range of concentration because of low molecular weight of their branches.

In Figure 5, the data of three-armed, starshaped P $\alpha$ MS S-22 in toluene and of S-22 and $\mathrm{S}-26$ in $\alpha-\mathrm{CN}$, as reported by Kajiura et al., ${ }^{10}$ are plotted in the form of $\log \eta_{\mathrm{R}}^{0} v s \cdot \log \left(C / C^{*}\right)$. It can be observed that all data compose a universal line as predicted from the scaling theory. Since the molecular weight dependence of $\eta^{0}$ of these samples is not known, however, we cannot compare the data with eq 1 . The solid line in Figure 5 was drawn assuming $a=3.7$. The dotted and broken lines denote $\eta_{\mathrm{R}}^{0}$ of linear $\mathrm{P} \alpha \mathrm{MS}$ in dilute and semidilute regions, respectively. ${ }^{1}$ The reason for the deviation of experimental data from the solid line in higher concentration (three highest data of $\mathrm{S}-22, C>0.25 \mathrm{~g} \mathrm{ml}^{-1}$ ) is the same as in Figure 4.

Since the experimental points for branched polymers are not numerous enough, the crossover between the dilute and semidilute regions and also between the semidilute and concentrated regions are not clear as in the case of linear polymers reported previously. ${ }^{1,2}$ However, it is clear that the features in Figs. $3-5$ are qualitatively the same as those for linear polymers.

From these results, therefore, we conclude that the semidilute region exists in viscosity behavior of branched polymers if the molecular weight of branches is high enough, and the scaling relationship (1) or (2) holds for star-shaped polymers if the number of branches is kept constant. That is, the experimental data of $\eta^{0}{ }_{\mathbf{R}}$ for branched polymers fit the theoretical lines (1) and (2) with $a=$ 4.3 or 3.7 (higher than 3.4 ) in the semidilute solutions. This means that the zero-shear viscosity of star-shaped polymers increases more sharply than that of linear polymers not only with molecular weight but also with polymer concentration. Moreover, the zeroshear viscosity of branched polymers was sometimes found to be higher than that of corresponding linear polymers with the same molecular weight. $^{11-13,17,19}$ This enhancement of $\eta^{0}$ due to branching may arise from the higher molecular weight dependence of $\eta^{0}$ of brached polymers.

\section{REFERENCES}

1. Y. Takahashi, Y. Isono, I. Noda, and M. Nagasawa, Macromolecules, 18, 1002 (1985).

2. Y. Takahashi, I. Noda, and M. Nagasawa, Macromolecules, in press.

3. P. G. de Gennes, "Scaling Concepts in Polymer Physics," Cornell University Press, Ithaca, New York, N. Y., 1979.

4. T. Kato, A. Itsubo, Y. Yamamoto, T. Fujimoto, and M. Nagasawa, Polym. J., 7, 123 (1975).

5. $\mathbf{Y}$. Isono, T. Fujimoto, $\mathrm{H}$. Kajiura, and $\mathbf{M}$. Nagasawa, Polym. J., 12, 369 (1980).

6. T. Fujimoto, H. Narukawa, and M. Nagasawa, Macromolecules, 3, 57 (1970).

7. T. Fujimoto, S. Tani, K. Takano, M. Ogawa, and M. Nagasawa, Macromolecules, 11, 673 (1978).

8. M. Sakai, T. Fujimoto, and M. Nagasawa, Macromolecules, 5, 786 (1972).

9. T. Kato, K. Miyaso, I. Noda, T. Fujimoto, and M. Nagasawa, Macromolecules, 3, 777 (1970).

10. H. Kajiura, Y. Ushiyama, T. Fujimoto, and M. Nagasawa, Macromolecules, 11, 894 (1978).

11. G. Kraus and J. T. Gruver, J. Polym. Sci., A, 3, 105 (1965).

12. G. Kraus and J. T. Gruver, J. Polym. Sci., Polym. Phys. Ed., 8, 305 (1970).

13. W. W. Graessley, T. Masuda, J. E. L. Roovers, and N. Hadjichristidis, Macromolecules, 9, 127 (1976).

14. T. Masuda, Y. Ohta, and S. Onogi, Macromolecules, 4, 763 (1971).

15. L. A Utracki and J. E. L. Roovers, Macromolecules, 6, 366 (1973).

16. Y. Isono, T. Fujimoto, H. Inagaki, M. Shishido, and M. Nagasawa, Polym. J., 12, 131 (1980).

17. G. C. Berry and T. G Fox, Adv. Polym. Sci., 5, 261 (1969).

18. Y. Higo, N. Ueno, and I. Noda, Polym. J., 15, 367 (1983).

19. W. W. Graessley, Acc. Chem. Res., 10, 332 (1977). 